\title{
ПСИХОФАРМАКОТЕРАПИЯ
}

\author{
УДК 616.895.87::615.21/.26
}

Для цитирования: Иванов М.В., Костерин Д.Н. Эффективность антипсихотической терапии препаратами пролонгированного действия у больных шизофренией в долгосрочной перспективе. Сибирский вестник психиатрии и наркологии. 2017; 3 (96): 84-91. https://doi.org/10.26617/1810-3111-2017-3(96)-84-91

\section{Эффрективность антипсихотической терапии препаратами пролонгированного действия}

\author{
Иванов М.В., Костерин Д.Н.
}

Национальный медицинский исследовательский центр психиатрии и неврологии имени В.М. Бехтерева 192019, Санкт-Петербург, ул. Бехтерева, д. 3

\section{PEЗЮME}

Цель исследования - оценка эффективности антипсихотической терапии препаратами пролонгированного действия больных параноидной шизофренией. Материал: проанализировано 75 случаев лечения больных параноидной шизофренией, получавших терапию LAI: палиперидоном пальмитатом (40 пациентов) или галоперидолом деканоатом (35 пациентов) 24 месяца после достижения ремиссии в 2013-2017 гг. В анализ также вошли данные 35 пациентов, продолжавших терапию LAI на базе СПб ГБУЗ Психоневрологический диспансер № 5 еще 12 месяцев. Задачи наблюдения: изучить динамику психического состояния больных на фоне приема LAI, сравнить эффективность и безопасность LAI первого и второго поколений, оценить качество ремиссии. Методы: клинико-динамический (Шкала позитивных и негативных синдромов, Шкала персонального и социального функционирования, Шкала общего клинического впечатления), статистический (метод дескриптивной статистики). Результаты: в рамках совершенствования терапевтических стратегий в качестве эффективного метода контроля приверженности терапии и заболевания следует использовать инъекционные антипсихотические препараты длительного действия (LAI). Представлены подходы к использованию LAI в современной психиатрии и данные наблюдения результатов применения LAI первого и второго поколений в течение 24 месяцев в рутинной клинической практике. Своевременное вмешательство и непрерывность лечения имеет решающее значение для достижения долгосрочной ремиссии, предотвращения злокачественного течения заболевания, снижения издержек и бремени болезни.

Ключевые слова: шизофрения, ремиссия, инъекционные антипсихотики длительного действия (LAI), приверженность к терапии, палиперидон пальмитат, галоперидол деканоат.

\section{ВВЕДЕНИЕ}

Шизофрения - изнурительное хроническое психическое заболевание, одно из 20 самых распространенных причин инвалидности во всем мире, что связано с высокой стоимостью лечения $[1,2]$. Экономическое бремя шизофрении в США в 2013 г. оценивалось в $\$ 155,7$ млрд, четверть из которых составили прямые медицинские расходы [2]. Суммарное бремя шизофрении в РФ в 2009 г. составило 196,7 млрд рублей, в том числе 54 млрд рублей (27,5\% бремени шизофрении) приходилось на прямые медицинские затраты и $85 \%$ - на больничное лечение [3]. Высокая стоимость рецидива шизофрении сопряжена с затратами на стационарное лечение и с повторными госпитализациями. В США и большинстве европейских стран прямые затраты на лечение рецидива шизофрении втрое, а иногда в 2-4 раза выше, чем стоимость поддержания ремиссии у того же пациента в течение 3 лет. Результаты фармакоэкономических расчетов оказания помощи пациентам с шизофренией показывают, что улучшение комплаенса может сократить число госпитализаций на $12 \%$ и их длительность на $13 \%$, что позволит снизить «предотвратимые затраты» на \$106 млн [3]. Высокое экономическое бремя заболевания в том числе связано и с тем, что болезнь затрагивает молодую работоспособную популяцию населения, что приводит к быстрому регрессу профессионального и социального функционирования, инвалидности. Социальная значимость проблемы данного заболевания затрагивает не только самих пациентов, но и общество в целом. У пациентов, страдающих психическими заболеваниями, обнаруживается нарушение социального функционирования в разных сфеpax жизни: снижение социально-трудового статуса и социальных контактов, отсутствие семьи, ухудшение материально-бытового положения [4].

Не смотря на то что антипсихотическая (АП) терапия является эффективным инструментом в лечении симптомов шизофрении и предотвращении рецидива, проблема сохранения приверженности терапии сохраняет свою актуальность [5]. Недостаточный контроль заболевания и множественные рецидивы имеют прямую зависимость с прогнозом социального функционирования и снижением чувствительности к антипсихотической терапии [6], а также способность вызвать цикл экономических, социальных и правовых проблем, создающих дополнительные препятствия для эффективного лечения [5]. Поэтому лечение пациентов на ранних стадиях заболевания, когда они могут быть наиболее поддающимися лечению, имеет первостепенное значение для минимизации риска последствий болезни в долгосрочной перспективе [7]. 
Связь между приверженностью к терапии и контролем заболевания была неоднократно доказана и продемонстрирована. Так, метаанализ 65 клинических исследований у пациентов с шизофренией, стабилизированных на антипсихотических препаратах, которые были рандомизированы для продолжения лечения антипсихотическими препаратами или плацебо, показал, что лечение антипсихотиками значительно снижает частоту рецидивов [8]. Другой метаанализ 6 плацебо-контролируемых рандомизированных клинических исследований у пациентов с острой шизофренией, получавших антипсихотические препараты, продемонстрировал значительное улучшение положительных и отрицательных симптомов в течение 6 недель с пропорционально возрастающим эффектом лечения у лиц с более тяжелыми симптомами на исходном уровне [9].

Однако лекарственный препарат эффективен только тогда, когда он принят, а несоблюдение приверженности лечению является серьезным препятствием для эффективного лечения пациентов с шизофренией. Выявлена закономерность в приверженности лечению в зависимости от длительности приема терапии: приверженность терапии уменьшается с увеличением длительности периода лечения. Так, 4-летнее ретроспективное кросс-секционное исследование большой когорты пациентов с шизофренией Департамента по делам ветеранов США показало, что $36 \%$ пациентов испытывали проблемы с приверженностью терапии ежегодно и $61 \%$ пациентов нарушали режим лечения в течение 4-летнего периода [10].

Постоянное соблюдение приема антипсихотических препаратов имеет решающее значение для оптимальных результатов лечения у пациентов с шизофренией. Прерывание лечения всего в течение $1-$ 10 дней повышает риски обострения заболевания и связанной с этим госпитализации пациентов [11]. Помимо госпитализации, несоблюдение терапии связано с увеличением финансового, социального, профессионального бремени заболевания: рост использования служб неотложной психиатрической помощи, насилия, арестов, попыток самоубийства, снижение социального и профессионального функционирования и качества жизни [12]. Очевидно, что вмешательства, направленные на улучшение приверженности терапии, способны изменить ситуацию в каждой из сфер, уменьшить бремя заболевания.

Хотя детерминанты несоблюдения терапии многочисленны и сложны, к одним из важных факторов, влияющих на комплаентность пациентов, относят факторы, связанные с лечением, например, выбор АП терапии и частоту дозирования [13]. Так, инъекционные антипсихотические препараты длительного действия (LAI) требуется вводить реже (1 раз в 1-12 недель) по сравнению с ежедневным приемом пероральных форм препаратов. Имеющиеся в настоящее время данные свидетельствуют о том, что для пациентов с шизофренией использование LAI второго поколения в целом связано с более низкими показа- телями рецидива заболевания и снижением смертности у взрослых по сравнению с атипичными антипсихотическими препаратами для перорального приема, что, вероятно, связано с увеличением приверженности к лечению $[14,15]$. Инъекционные антипсихотики длительного действия являются важной альтернативой пероральным препаратам, особенно выгодной в контексте управления приверженности терапии. Положительным моментом стало то, что генерация антипсихотиков нового поколения, включая рисперидон, оланзапин, палиперидон и арипипразол, стала доступна в виде препаратов длительного действия. На сегодняшний день все LAI нового (второго) поколения продемонстрировали статистически и клинически значимое снижение частоты развития рецидивов по сравнению с плацебо. LAI нового поколения оказались сопоставимы с их пероральными формами в отношении безопасности и переносимости. Существует мало свидетельств различий в эффективности между доступными LAI [16], однако различия, с точки зрения фармакодинамических и фармакокинетических профилей, интервала между инъекциями, стоимости, требований к сопутствующей терапии, а также спектра побочных эффектов, стоит учитывать при выборе LAI для лечения отдельных пациентов. Авторами приводится всё больше свидетельств, указывающих на необходимость приоритетного использования LAI у пациентов после первого эпизода и заключенных, страдающих шизофренией.

Далее приведены данные об опыте применения LAI при шизофрении, обсуждаются текущие проблемы с клинической и методологической точек зрения.

LAI: рекомендациии по использованию

Текущие рекомендации [17] предлагают рассматривать LAI в качестве препаратов первого выбора для поддерживающей терапии шизофрении среди других доступных вариантов в лечении или когда необходимо улучшить приверженность к терапии. LAI также следует рассмотреть в качестве препарата первого выбора для терапии шизофрении, если у пациента имелись данные о несоблюдении терапии в анамнезе или выявлен нерегулярный прием поддерживающей терапии [17]. При этом многие эксперты считают, что эти действующие рекомендации являются слишком ограниченными и показания к назначению LAI в лечении шизофрении должны быть расширены [18]. С увеличением доступности LAI нового поколения для наиболее эффективного их применения международные рекомендации должны учитывать такие факторы, как стадию заболевания и популяцию пациентов, которая потенциально может извлечь наибольшую выгоду из лечения подобными препаратами.

Удивительный факт был обнаружен по материалам опросов, проведенных в нескольких странах Западной Европы, по опыту применения LAI [19]: крайне бедный опыт использования LAI даже у пациентов, которые могли бы извлечь из этого выгоду. 
Использование LAI рассматривалось как старый и принудительный способ лечения, который следует использовать для пациентов с плохой приверженностью к терапии и как связанный с уменьшением вовлеченности медицинского персонала в уход за пациентами [19]. Некоторые психиатры называли их средством «последней надежды», которое должно использоваться, когда все другие фармакологические методы лечения потерпели неудачу, или как препараты резерва для пациентов, которые перенесли многочисленные обострения заболевания. Это объясняется большим числом недоразумений и предрассудков, распространенных среди врачей, пациентов и лиц, осуществляющих уход за пациентами, страдающими шизофренией. Высокая стоимость LAI нового поколения представляет собой еще одно серьезное препятствие для широкого назначения этих препаратов. Тем не менее недавние фармакоэкономические исследования показали, что применение LAI второго поколения способно стать превосходной терапевтической стратегией с экономической точки зрения, принимая в расчет не только прямые, но и косвенные затраты на уход за пациентами [20].

Большинство специалистов сходятся в единодушном мнении, что оптимальное использование LAI второго поколения требует радикального изменения общего подхода к лечению депонированными антипсихотическими препаратами [18]. По мнению ряда исследователей, LAI второго поколения следует рассматривать в качестве потенциальных препаратов первого выбора, которые должны быть использованы для пациентов, нуждающихся в длительном лечении, а не только в случаях с низкой приверженностью к терапии [18]. Эффективная фармакологическая поддерживающая терапия является отправной точкой для успеха программ мультимодального лечения и реабилитации для людей, страдающих шизофренией. Принято считать, что лечение пероральными антипсихотиками следует начинать как можно скорее у пациентов с впервые диагностированной шизофренией [17]. Несмотря на то что данные об эффективности применения LAI второго поколения в этой области все еще ограничены, можно предположить, что они по меньшей мере столь же предпочтительны, как и пероральные антипсихотики. Для пациентов с обострением шизофрении, которым согласно действующим рекомендациям предлагается лечение пероральными антипсихотиками, следует рассмотреть назначение LAI второго поколения, когда эти обострения обусловлены предшествующим несоблюдением терапии и низкой приверженностью к терапии в анамнезе [21]. Следует принимать во внимание, что переход от пероральных антипсихотиков к LAI второго поколения требует определенных стратегий, чтобы поддержать или улучшить терапевтический эффект и минимизировать влияние потенциального холинергического или гистаминергического рикошета [21].
Основной целью лечения больных шизофренией является достижение ремиссии заболевания. Раннее вмешательство и непрерывность терапии имеют решающее значение для достижения долгосрочной ремиссии, предотвращения злокачественного течения заболевания и снижения издержек и бремени болезни. Традиционно LAI были зарезервированы для пациентов, не являющихся приверженцами, которые перенесли множественные эпизоды обострения заболевания. Доступность LAI второго поколения с лучшей переносимостью, чем LAI первого поколения с точки зрения развития экстрапирамидных побочных эффектов, дает возможность широко применять такое лечение у молодых пациентов на начальных этапах шизофрении. Это особенно актуально с учетом рисков рецидива после прекращения лечения и разрушительных последствий рецидива. Дальнейшее образование врачей и просвещение пациентов необходимо для того, чтобы рассмотреть LAI c новой точки зрения: больше не как лекарственные средства «последней надежды», а как первый шаг для достижения непрерывности лечения и клинической ремиссии [21]. При выборе тактики терапии следует уделять внимание не только антипсихотическому эффекту, но и другим клиническим, психологическим, социальным и фармакоэкономическим характеристикам, а также оценке их сочетания в каждом отдельном случае. Сохраняется актуальность изучения вопросов клинических особенностей течения ремиссии, механизмов развития, систематизации, прогноза, проявления и коррекции возможных побочных эффектов, влияющих на дальнейший исход заболевания [22, 23]. Появление LAI второго поколения увеличило портфель средств терапии, доступных для создания индивидуального подхода к лечению шизофрении - давно забытого, но ключевого аспекта в лечении пациентов с психическими заболеваниями [21].

Цель исследования - оценка эффективности антипсихотической терапии препаратами пролонгированного действия больных параноидной шизофренией.

\section{МАТЕРИАЛЫ И МЕТОДЫ}

LAI: результаты 24 месячного наблюдения

Для изучения результатов применения LAI было проанализировано 75 случаев лечения пациентов с диагнозом параноидной формы шизофрении (F20.0 по МКБ-10), получавших терапию LAI в рутинной клинической практике: палиперидоном пальмитатом (40 пациентов, далее - группа ПП) или галоперидолом деканоатом (35 пациентов, далее - группа ГД) в течение 24 месяцев после достижения ремиссии в 2013-2017 гг. В анализ также были включены данные 35 пациентов, продолжавших терапию LAI на базе СПб ГБУЗ Психоневрологический диспансер № 5 [24] еще на протяжении 12 месяцев. В задачи наблюдения входило: изучить динамику психического состояния больных на фоне приема LAI, сравнить эффективность и безопасность LAI первого и второго поколений, оценить качество ремиссии. 
Далее представлены данные, полученные по результатам наблюдения за пациентами, получавшими терапию LAI первой и второй генерации на протяжении 24 месяцев в рутинной клинической практике.

Методы наблюдения

Для оценки результатов проводимой терапии использовались данные шкальной оценки: Шкала позитивных и негативных синдромов (PANSS), Шкала персонального и социального функционирования (PSP), Шкала общего клинического впечатления (CGI-S). Полученные данные обработаны с использованием программы Windows Microsoft Office Excel 2007 методом дескриптивной статистики.

\section{РЕЗУЛЬТАТЫ И ОБСУЖДЕНИЕ}

На исходном уровне пациенты были сопоставимы по возрасту $(43,8 \pm 10,3$ года - в группе ПП, $44,6 \pm 10,3$ года - в группе ГД), по среднему возрасту начала заболевания $(23,5 \pm 7,9$ и $28,45 \pm 3,5$ года группы ПП и ГД соответственно) и длительности

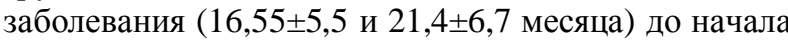
терапии LAI. Семейный статус пациентов также не различался: в браке состояли только 27,5\% и $34 \%$ пациентов из групп ПП и ГД соответственно. Группа ПП имела исходное преимущество в профессиональном статусе: до перевода на терапию LAI 75\% пациентов продолжали работать и не имели инвалидности, у 50\% имелось снижение уровня профессионального функционирования. Профессиональный статус пациентов группы ГД отличался в худшую сторону: 80\% пациентов не работали в связи с заболеванием и практически $88,5 \%$ имели инвалидность. По уровню социального функционирования показатели исходного уровня были в пользу пациентов, переведенных на терапию галоперидолом деканоатом: адекватное отношение близких к болезни и наличие хороших отношений с близкими наблюдалось более чем в половине случаев, но в то же время значительное ограничение круга социальных интересов у 88,5\% пациентов. В группе пациентов, которым был назначен палиперидон пальмитат, исходно преобладало неадекватное отношение близких к болезни, хорошие и формальные отношения с близкими встречались в равном соотношении, у подавляющего большинства пациентов социальные контакты были ограничены, отмечалось сужение или утрата социальных интересов.

Частота госпитализаций пациентов до их перевода на терапию LAI практически не различалась: $50,0 \%$ и 48,5\% случаев в группах ПП и ГД соответственно. Оказался схожим и тип течения заболевания: в $75,0 \%$ и $62,8 \%$ случаев установлен периодический приступообразно-прогредиентный тип течения, средняя продолжительность ремиссии до перевода на терапию LAI составляла $11,7 \pm 1,7$ месяца

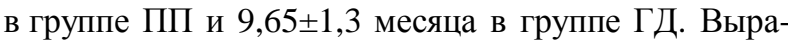
женность психопатологической симптоматики обследованных была сопоставима и соответствовала уровню «умеренного психического расстройства»

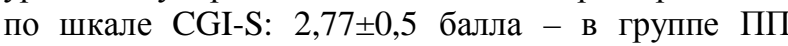

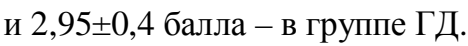

При этом выраженность психопатологической симптоматики по шкале PANSS исходно была незначительно меньше в группе ПП (61,2士7,9 балла),

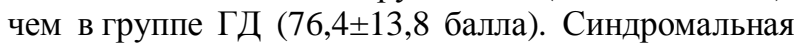
структура заболевания характеризовалась преобладанием параноидного синдрома в обеих группах, с преобладанием аффективной составляющей у пациентов группы ПП и галлюцинаторной симптоматики у пациентов, переведенных на терапию галоперидолом деканоатом.

Результаты проводимой терапии LAI продемонстрировали положительное влияние на течение болезни у обследованных пациентов: в течение 24 месяцев наблюдения 47,5\% пациентов, получавших палиперидон пальмитат, и у 43 \% пациентов, получавших галоперидол деканоат, все они не нуждались в назначении дополнительной антипсихотической терапии. По окончании 24 месяцев наблюдения была зарегистрирована положительная динамика психического состояния пациентов вне зависимости от вида LAI по шкале PANSS (рис.1): снижение показателя по PANSS на 4\% в группе ПП и на $9 \%$ в группе ГД.

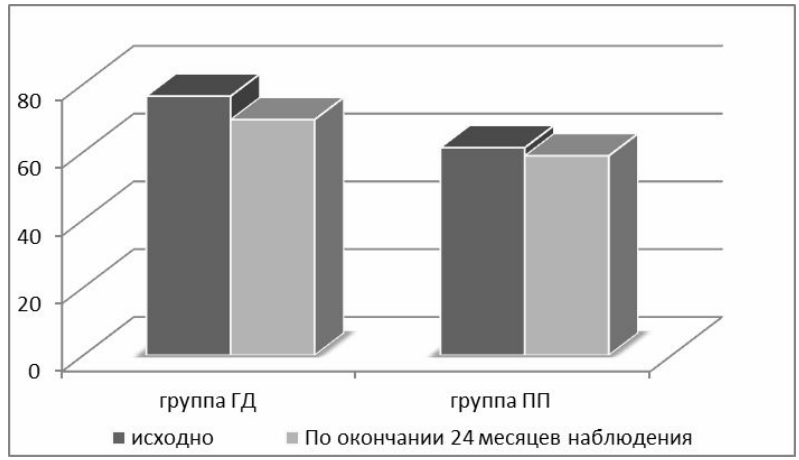

Рисунок 1. Динамика психического состояния по PANSS

Неполное купирование психопатологической симптоматики отмечено в 7,5\% в группе ПП, в $14,2 \%$ в группе ГД (р>0,05), что, видимо, связано с исходными различиями пациентов по этим характеристикам. Показатели шкалы CGI значимо не изменились: $3,01 \pm 2,6$ и 2,3 $\pm 0,6$ балла в группах ГД и ПП, что подтвердило стабильное психическое состояние пациентов обеих групп, наличие ремиссии. Положительная динамика выявлена по показателям социального функционирования у пациентов группы ПП (рис. 2): социальное функционирование по PSP увеличилось на $11 \%$ по сравнению с разницей в 6\% (p<0,05) у пациентов, получавших галоперидол деканоат. Уровень социального функционирования

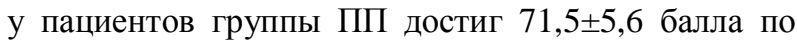
PSP (степень легкого затруднения функционирования в основных областях). У пациентов, получавших галоперидол деканоат, уровень социального функционирования оказался достоверно ниже и со-

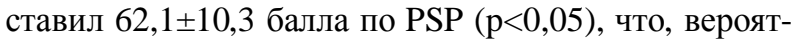
но, связано с наличием более выраженной формы социальной и профессиональной дезадаптации. 


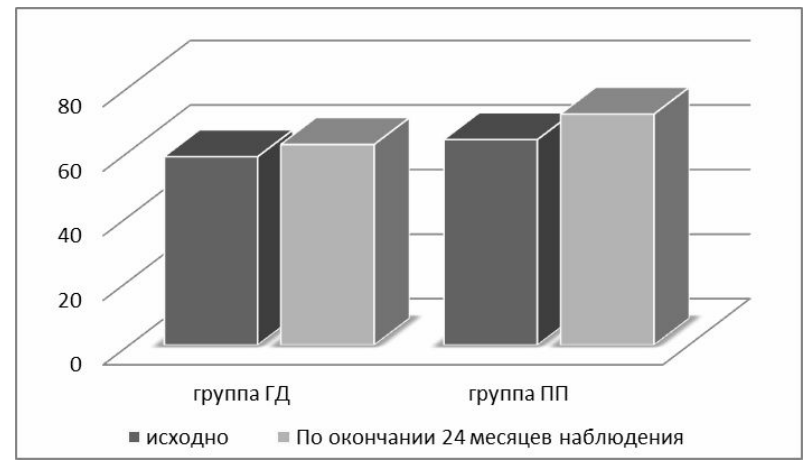

Рисунок 2. Динамика социального функционирования по PSP

При этом следует отметить, что палиперидон пальмитат назначался более социально адаптированным больным, что, вероятно, и повлияло на более значимое улучшение их социального функционирования в дальнейшем по сравнению с пациентами, получавшими галоперидол деканоат.

Установлено, что перевод на терапию LAI способствовал повышению качества и продолжительности ремиссии по сравнению с исходным уровнем, в том числе у пациентов, перенесших обострения заболевания в течение периода наблюдения (рис. 3).

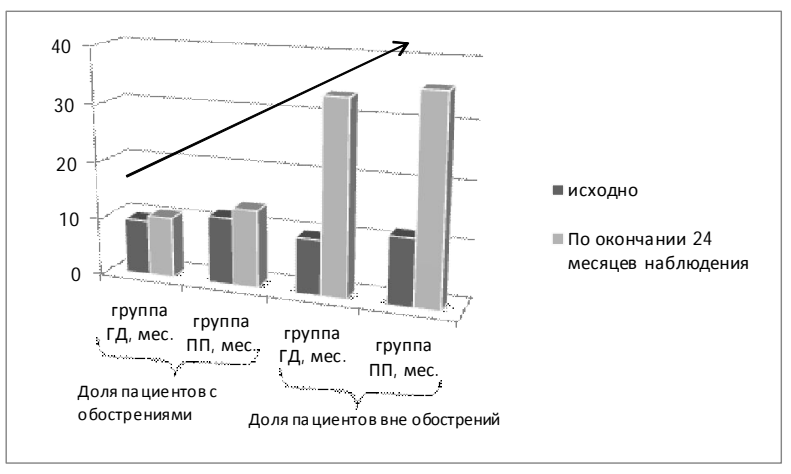

Рисунок 3. Средняя продолжительность ремиссии по истечении 24 месяцев наблюдения

В течение всего периода наблюдения за пациентами, получавшими палиперидон пальмитат, не было зарегистрировано обострений, требующих госпитализации в психиатрический стационар (в 45\% случаев зафиксированы обострения, сопровождавшиеся усилением внебольничной психиатрической помощи). В то же время пациентам, получавшим галоперидол деканоат, госпитализация потребовалась в 28,6\% случаев (из 57 \% случаев обострения заболевания). Таким образом, стабильная ремиссия на протяжении всего периода наблюдения выявлена у 55\% пациентов в группе ПП и у $43 \%$ пациентов в группе ГД, что является хорошим и качественным показателем результатов проводимой терапии. На рисунке 3 представлена динамика средней продолжительности ремиссии для пациентов, перенесших обострения на протяжении 24 месяцев наблюдения, и для пациентов, сохранявших стабильную ремиссию на протяжении этого срока.
В течение всего периода наблюдения большинству пациентов, получавших палиперидон пальмитат, требовалось проведение коррекции дозы (73\% случаев, в $12,5 \%$ из которых проводилось снижение дозы); при этом 67\% пациентов, получавших галоперидол деканоат, не нуждались в коррекции дозы.

По профилю безопасности препараты первой и второй генераций LAI оказались схожими: побочные эффекты легкой степени тяжести со стороны психической, неврологической, эндокринной, соматической систем наблюдались примерно с одинаковой частотой $-45 \%$ и $46 \%$ в группах ПП и ГД соответственно. Необходимость присоединения антихолинергических средств чаще требовалась пациентам, получавшим LAI первого поколения (10\% и $14 \%$ случаев), как и назначение транквилизаторов для коррекции симптомов ажитации (30,0\% и $57,1 \%$ случаев), что, как известно, связано с побочными эффектами LAI первого поколения. Частота назначения сопутствующей антидепрессивной терапии в группах достоверно не различалась (45\% и $51 \%$ случаев).

Таким образом, представленные результаты наблюдения за эффектами терапии LAI свидетельствуют о положительном влиянии данных препаратов на течение болезни. Применение инъекционных антипсихотических препаратов пролонгированного действия способствовало улучшению психического статуса пациентов, сохранению приверженности терапии и улучшению качества ремиссии заболевания, снижению экономического бремени болезни. Безусловно, данные результаты ограничены небольшой выборкой наблюдений (75 случаев) и для экстраполяции полученных данных на более широкую популяцию пациентов изучение эффектов терапии LAI первой и второй генераций должно быть продолжено на более массивной исследовательской выборке пациентов.

\section{ЗАКЮЧЕНИЕ}

Результаты наблюдения за пациентами, получавшими терапию LAI в течение 2 лет, подтвердили, что терапия пролонгированными антипсихотиками как первой, так и второй генерации может обеспечить ремиссию в большинстве случаев. Создание условий для непрерывности антипсихотической терапии может сыграть определяющую роль в улучшении результатов лечения шизофрении [25]. Полученные данные, с учетом современных тенденций в использовании LAI, вселяют оптимизм и создают благоприятную почву для более широкого применения этих препаратов в рутинной клинической практике. Депонированные препараты считаются средством выбора для длительной поддерживающей терапии шизофрении, поскольку позволяют улучшить приверженность лечению и социальную адаптацию пациентов [26]. Инъекционные антипсихотики пролонгированного действия упрощают схему и обеспечивают непрерывность терапии, способствуют улучшению психического состояния пациентов после стабилизации заболевания [27]. 
Инъекционные пролонгированные антипсихотики второй генерации сочетают преимущества атипичных антипсихотиков с непрерывностью воздействия пролонгированных форм и способны стать препаратами выбора для длительной, надёжной и эффективной противорецидивной терапии шизофрении не только у молодых пациентов [28, 29].

Накопление практического материала и дальнейшее наблюдение за пациентами, получающими LAI первого и второго поколений, будет способствовать улучшению имеющихся стандартов лечения и созданию персонализированных подходов к терапии больных, страдающих шизофренией.

\section{КОНФЛИКТ ИНТЕРЕСОВ}

Авторы заявляют об отсутствии конфликта интересов в связи с публикацией данной статьи.

\section{ИСТОЧНИК ФИНАНСИРОВАНИЯ}

Авторы заявляют об отсутствии финансирования при проведении исследования.

\section{СООТВЕТСТВИЕ ПРИНЦИПАМ ЭТИКИ}

Работа соответствует этическим стандартам, разработанным согласно принципам исследовательской этики Хельсинской декларации Всемирной медицинской ассоциации (протокол заседания ЛЭК «НМИЦ ПН им. В.М. Бехтерева» № 6 от 22.06.2017 г.).

\section{ЛИТЕРАТУРА}

1. Whiteford H.A., Degenhardt L., Rehm J, Baxter A.J., Ferrari A.J., Erskine H.E. et al. Global burden of disease attributable to mental and substance use disorders: Findings from the Global Burden of Disease Study, 2010. Lancet. 2013; 382 (9904): 1575-1586.

2. Cloutier M., Sanon Aigbogun M., Guerin A., Nitulescu R., Ramanakumar A.V., Kamat S.A. et al. The economic burden of schizophrenia in the United States in 2013. J. Clin. Psychiatry. 2016

3. Любов Е.Б., Ястребов В.С., Шевченко Л.С., Чапурин С.А. и др. Экономическое бремя шизофрении в России. Сочиальная и клиническая психиатрия. 2012; 22 (3): 36-42.

4. Рахмазова Л.Д., Агарков А.А., Васильева Н.А., Петрачков И.В., Погорелова Т.В. Клинико-социальная адаптация пациентов с психозами и состояниями слабоумия. Сибирский вестник психиатрии и наркологии. 2014; 2 (83): 9-13.

5. Higashi K., Medic G., Littlewood K.J., Diez T., Granström O., De Hert M. Medication adherence in schizophrenia: factors influencing adherence and consequences of nonadherence, a systematic literature review. Ther. Adv. Psychopharmacol. 2013; 3 (4):200-218

6. Jäger M., Riedel M., Messer T., Laux G., Pfeiffer H., Naber D. et al. Psychopathological characteristics and treatment response of first episode compared with multiple episode schizophrenic disorders. Eur. Arch. Psychiatry. Clin. Neurosci. 2007; 257 (1): 47-53.

7. McGorry P.D., Killackey E., Yung A. Early intervention in psychosis: concepts, evidence and future directions. World Psychiatry. 2008; 7 (3): 148-156.

8. Leucht S., Cipriani A., Spineli L. et al. Comparative efficacy and tolerability of 15 antipsychotic drugs in schizophrenia: a multipletreatments meta-analysis. Lancet. 2013; 382 (9896): 951-962.

9. Mattila T., Wohlfarth T., Koeter M. et al. Geographic variation in efficacy of atypical antipsychotics for the acute treatment of schizophrenia - an individual patient data meta-analysis. Eur. Neuropsychopharmacol. 2014; 24 (7): 1066-1077.

10. Slomko H., Heo H.J., Einstein F.H. Minireview: epigenetics of obesity and diabetes in humans. Endocrinology. 2012; 153 (3): 1025-1030.

11. Choi J.H., Yoo H.W. Control of puberty: genetics, endocrinology, and environment. Curr. Opin. Endocrinol. Diabetes Obes. 2013 20 (1): 62-68.

12. Zandi P.P., Judy J.T. The promise and reality of pharmacogenetics in psychiatry. Clin. Lab. Med. 2010; 30 (4): 931-974.

13. Medic G., Higashi K., Littlewood K.J., Diez T., Granstrom O.,
Kahn R.S. Dosing frequency and adherence in chronic psychiatric disease: Systematic review and meta-analysis. Neuropsychiatr. Dis. Treat. 2012; 9: 119-131.

14. Marcus S.C., Zummo J., Pettit A.R., Stoddard J., Doshi J.A. Antipsychotic Adherence and Rehospitalization in Schizophrenia Patients Receiving Oral Versus Long-Acting Injectable Antipsychotics Following Hospital Discharge. J. Manag. Care Spec. Pharm. 2015; 21 (9): 754-769.

15. Lafeuille M.-H., Grittner A.M., Fortier J., Muser E., Fasteneau J., Duh M.S. et al. Comparison of rehospitalization rates and associated costs among patients with schizophrenia receiving paliperidone palmitate or oral antipsychotics. Am. J. Health Pharm. 2015; $72(5)$ : 378-389.

16. Correll C.U., Citrome L., Haddad P.M., Lauriello J., Olfson M., Calloway S.M. et al. The use of long-acting injectable antipsychotics in schizophrenia: evaluating the evidence. J. Clin. Psychiatry. 2016; 77 (suppl 3): 1-24.

17. Jääskeläinen E., Juola P., Hirvonen N. et al. A systematic review and meta-analysis of recovery in schizophrenia. Schizophr. Bull. 2013; 39: 1296-306.

18. Kane J.M., Garcia-Ribera C. Clinical guideline recommendations for antipsychotic long-acting injections. Br. J. Psychiatry. 2009; 52 (Suppl): S63-67.

19. Besenius C., Clark-Carter D., Nolan P. Health professionals' attitudes to depot injection antipsychotic medication: a systematic review. J. Psychiatr. Ment. Health Nurs. 2010; 17: 452-462.

20. Achilla E., McCrone P. The cost effectiveness of longacting/extended-release antipsychotics for the treatment of schizophrenia: a systematic review of economic evaluations. Appl. Health Econ. Health Policy. 2013; 11: 95-106.

21. Newcomer J.W., Weiden P.J., Buchanan R.W. Switching antipsychotic medications to reduce adverse event burden in schizophrenia: establishing evidence-based practice. J. Clin. Psychiatry. 2013; 74: 1108-1120.

22. Незнанов Н.Г., Масловский С.Ю., Иванов М.В. Качество жизни больных шизофренией в процессе противорецидивной терапии. Психиатрия и психофармакотерапия. 2004; 6 (5): 213-215.

23. Потапов А.В. Стандартизированные клинико-функциональные критерии терапевтической ремиссии при шизофрении (популяционное, фармакоэпидемиологическое и фармакотерапевтическое исследование): автореф. дис. ... к.м.н. М., 2010: 24.

24. Петрова Н.Н., Иванов М.В., Костерин Д.Н., Серазетдинова В.С., Савельева О.В. Характеристика ремиссии у больных шизофренией на фоне лечения антипсихотическими препаратами пролонгированного действия. Психиатрия и психофармакотерапия им. П.Б. Ганнуикина. 2015; 05-06: 20-25.

25. Данилов Д.С. Комплаенс в медицине и методы его оптимизации (клинические, психологические и психотерапевтические аспекты). Психиатрия и психофармакотерапия. 2008; 10 (1): $13-20$.

26. Kirson N.Y., Weiden P.J., Yermakov S. et al. Efficacy and effectiveness of depot versus oral antipsychotics in schizophrenia: synthesizing results across different research designs. J. Clin. Psychiatry. 2013; 74 (6): 568-575.

27. Руководство по биологической терапии шизофрении Международной Федерации Обществ Биологической Психиатрии (WFSBP). Обновление от 2012 года. Современная терапия психических расстройств. 2014; 2: 3-40.

28. Петрова Н.Н., Серазетдинова Л.Г., Баранов С.Н., Вишневская О.А., Малеваная О.В., Московцева О.Р. Палиперидона пальмитат в решении актуальных проблем лечения больных шизофренией. Социальная и клиническая психиатрия. 2013; 23 (1): 73-78.

29. Петрова Н.Н., Кучер Е.О. Препараты пролонгированного действия в поддерживающей терапии больных с первым психотическим эпизодом. Социальная и клиническая психиатрия. 2014; 3: 67-72.

Поступила в редакцию 26.05.2017 Утверждена к печати 26.06.2017 
Иванов Михаил Владимирович, д.м.н., проф., руководитель 1-го отделения биологической терапии психически больных. Костерин Дмитрий Николаевич, к.м.н., научный сотрудник 1-го отделения биологической терапии психически больных.

Иванов Михаил Владимирович, profmikhailivanov@gmail.com

УДК 616.895.87::615.21/.26

For citation: Ivanov M. V., Kosterin D.N. Long-term outcomes of long-acting injection antipsychotic treatment in patients with schizophrenia. Siberian Herald of Psychiatry and Addiction Psychiatry. 2017; 3 (96): 84-91. https://doi.org/10.26617/18103111-2017-3(96)-84-91

\title{
Long-term outcomes of long-acting injection antipsychotic treatment in patients with schizophrenia
}

\author{
Ivanov M. V., Kosterin D.N.
}

St. Petersburg V.M. Bekhterev Psychoneurological Research Institute

Bekhterev Street 3, 192019, St. Petersburg, Russian Federation

\section{ABSTRACT}

Objective of the study: to evaluate efficiency of antipsychotic therapy with long-acting agents for patients with paranoid schizophrenia. Material: 75 cases of patients with paranoid schizophrenia under therapy with LAI were analyzed: paliperidone palmitate $(n=40)$ or haloperidol decanoate $(n=35)$ for 24 months after achieving remission in 2013-2017. The analysis included also data of 35 patients who continued the LAI therapy at St. Petersburg Psychoneurological Dispensary no. 512 months more. Tasks of observation: to study dynamics of mental health of patients against the background of intake of LAI, to compare efficiency and safety of LAI of the first and second generations, to evaluate quality of remission. Methods: clinical-dynamic (PANSS, PSP, CGI), statistical (method of descriptive statistics). Results: In order to improve therapeutic strategies long-acting injection antipsychotics (LAI) should be used as an effective method of monitoring adherence and disease. There are approaches to the use of LAI in modern psychiatry and the data of the observation of the first and second generation LAI administration for 24 months in routine clinical practice. Well-timed intervention and continuity of treatment are decisive for achieving long-term remission, preventing a malicious course of the disease and reducing the costs and the burden of the disease.

Keywords: schizophrenia, remission, long-acting injection antipsychotic (LAI), adherence to therapy, paliperidone palmitate, haloperidol decanoate.

\section{REFERENCES}

1. Whiteford H.A., Degenhardt L., Rehm J, Baxter A.J., Ferrari A.J. Erskine H.E. et al. Global burden of disease attributable to mental and substance use disorders: Findings from the Global Burden of Disease Study, 2010. Lancet. 2013; 382 (9904): 1575-1586.

2. Cloutier M., Sanon Aigbogun M., Guerin A., Nitulescu R., Ramanakumar A.V., Kamat S.A. et al. The economic burden of schizophrenia in the United States in 2013. J. Clin. Psychiatry. 2016.

3. Lyubov E.B., Yastrebov V.S., Shevchenko L.S., Chapurin S.A. et al. Jekonomicheskoe bremja shizofrenii v Rossii [Economic burden of schizophrenia in Russia]. Social'naja i klinicheskaja psihiatrija [Social and Clinical Psychiatry]. 2012; 22 (3): 36-42 (in Russian).

4. Rakhmazova L.D., Agarkov A.A., Vasilieva N.A., Petrachkov I.V., Pogorelova T.V. Kliniko-social'naja adaptacija pacientov s psihozami i sostojanijami slaboumija [Clinical-social adaptation of patients with psychoses and states of dementia]. Sibirskij vestnik psihiatrii i narkologii - Siberian Herald of Psychiatry and Addiction Psychiatry. 2014; 2 (83): 9-13 (in Russian).

5. Higashi K., Medic G., Littlewood K.J., Diez T., Granström O., De Hert M. Medication adherence in schizophrenia: factors influencing adherence and consequences of nonadherence, a systematic literature review. Ther. Adv. Psychopharmacol. 2013; 3 (4):200-218.

6. Jäger M., Riedel M., Messer T., Laux G., Pfeiffer H., Naber D. et al. Psychopathological characteristics and treatment response of first episode compared with multiple episode schizophrenic disorders. Eur. Arch. Psychiatry. Clin. Neurosci. 2007; 257 (1): 47-53.

7. McGorry P.D., Killackey E., Yung A. Early intervention in psychosis: concepts, evidence and future directions. World Psychiatry. 2008; 7 (3): 148-156.

8. Leucht S., Cipriani A., Spineli L. et al. Comparative efficacy and tolerability of 15 antipsychotic drugs in schizophrenia: a multipletreatments meta-analysis. Lancet. 2013; 382 (9896): 951-962.

9. Mattila T., Wohlfarth T., Koeter M. et al. Geographic variation in efficacy of atypical antipsychotics for the acute treatment of schizophrenia - an individual patient data meta-analysis. Eur. Neuropsychopharmacol. 2014; 24 (7): 1066-1077.

10. Slomko H., Heo H.J., Einstein F.H. Minireview: epigenetics of obesity and diabetes in humans. Endocrinology. 2012; 153 (3): 1025-1030.

11. Choi J.H., Yoo H.W. Control of puberty: genetics, endocrinology, and environment. Curr. Opin. Endocrinol. Diabetes Obes. 2013; 20 (1): 62-68.

12. Zandi P.P., Judy J.T. The promise and reality of pharmacogenetics in psychiatry. Clin. Lab. Med. 2010; 30 (4): 931-974.

13. Medic G., Higashi K., Littlewood K.J., Diez T., Granstrom O., Kahn R.S. Dosing frequency and adherence in chronic psychiatric disease: Systematic review and meta-analysis. Neuropsychiatr. Dis. Treat. 2012; 9: 119-131.

14. Marcus S.C., Zummo J., Pettit A.R., Stoddard J., Doshi J.A. Antipsychotic Adherence and Rehospitalization in Schizophrenia Patients Receiving Oral Versus Long-Acting Injectable Antipsychotics Following Hospital Discharge. J. Manag. Care Spec. Pharm. 2015; 21 (9): 754-769.

15. Lafeuille M.-H., Grittner A.M., Fortier J., Muser E., Fasteneau J., Duh M.S. et al. Comparison of rehospitalization rates and associated costs among patients with schizophrenia receiving paliperidone palmitate or oral antipsychotics. Am. J. Health Pharm. 2015; 72 (5): 378-389.

16. Correll C.U., Citrome L., Haddad P.M., Lauriello J., Olfson M., Calloway S.M. et al. The use of long-acting injectable antipsychotics in schizophrenia: evaluating the evidence. J. Clin. Psychiatry. 2016; 77 (suppl 3): 1-24.

17. Jääskeläinen E., Juola P., Hirvonen N. et al. A systematic review and meta-analysis of recovery in schizophrenia. Schizophr. Bull. 2013; 39: 1296-306.

18. Kane J.M., Garcia-Ribera C. Clinical guideline recommendations for antipsychotic long-acting injections. Br. J. Psychiatry. 2009; 52 (Suppl): S63-67.

19. Besenius C., Clark-Carter D., Nolan P. Health professionals' attitudes to depot injection antipsychotic medication: a systematic review. J. Psychiatr. Ment. Health Nurs. 2010; 17: 452-462.

20. Achilla E., McCrone P. The cost effectiveness of longacting/extended-release antipsychotics for the treatment of schizophrenia: a systematic review of economic evaluations. Appl. Health Econ. Health Policy. 2013; 11: 95-106. 
21. Newcomer J.W., Weiden P.J., Buchanan R.W. Switching antipsychotic medications to reduce adverse event burden in schizophrenia: establishing evidence-based practice. J. Clin. Psychiatry. 2013; 74: 1108-1120.

22. Neznanov N.G., Maslovsky S.Yu., Ivanov M.V. Kachestvo zhizni bol'nyh shizofreniej $\mathrm{v}$ processe protivorecidivnoj terapii [Quality of life of schizophrenic patients in the process of anti-relapse therapy]. Psihiatrija i psihofarmakoterapija - Psychiatry and Psychopharmacotherapy. 2004; 6 (5): 213-215 (in Russian).

23. Potapov A.V. Standartizirovannye kliniko-funkcional'nye kriterii terapevticheskoj remissii pri shizofrenii (populjacionnoe, farmakojepidemiologicheskoe i farmakoterapevticheskoe issledovanie) [Standardized clinical-functional criteria of therapeutic remission in schizophrenia (population, pharmaco-epidemiological and pharmacotherapeutic study)]. Avtoref. dis. ... k.m.n. - Abstract of PhD thesis. M., 2010: 24 (in Russian).

24. Petrova N.N., Ivanov M.V., Kosterin D.N., Serazetdinova V.S., Savelyeva O.V. Kharakteristika remissii u bol'nykh shizofreniey na fone lecheniya antipsikhoticheskimi preparatami prolongirovannogo deystviya [Characteristics of remission in patients with schizophrenia during treatment with antipsychotic drugs prolonged actionя]. Psihiatrija i psihofarmakoterapija - Psychiatry and Psychopharmacotherapy. 2015; 05-06: 20-25 (in Russian).

25. Danilov D.S. Komplaens v meditsine i metody ego optimizatsii (klinicheskie, psikhologicheskie i psikhoterapevticheskie aspekty) [Compliance in medicine and methods of its optimization (clinical, psychological and psychotherapeutic aspects)]. Psihiatrija i psi- hofarmakoterapija - Psychiatry and Psychopharmacotherapy. 2008; 10 (1): 13-20 (in Russian).

26. Kirson N.Y., Weiden P.J., Yermakov S. et al. Efficacy and effectiveness of depot versus oral antipsychotics in schizophrenia: synthesizing results across different research designs. J. Clin. Psychiatry. 2013; 74 (6): 568-575.

27. Rukovodstvo po biologicheskoy terapii shizofrenii Mezhdunarodnoy Federatsii Obshchestv Biologicheskoy Psikhiatrii (WFSBP). Obnovlenie ot 2012 goda [WFSBP Guidelines on biological therapy of schizophrenia. Updated in 2012]. Sovremennaya terapiya psikhicheskikh rasstroystv - Modern Therapy of Mental Disorders. 2014; 2: 3-40 (in Russian).

28. Petrova N.N., Serazetdinova L.G., Baranov S.N., Vishnevskaya O.A., Malyovanaya O.V., Moskovtseva O.R. Paliperidona pal'mitat $\mathrm{v}$ reshenii aktual'nykh problem lecheniya bol'nykh shizofreniey [Paliperidone palmitate in resolving relevant problems of therapy of schizophrenic patients]. Social'naja i klinicheskaja psihiatrija [Social and Clinical Psychiatry]. 2013; 23 (1): 73-78 (in Russian).

29. Petrova N.N., Kucher E.O. Preparaty prolongirovannogo deystviya $\mathrm{v}$ podderzhivayushchey terapii bol'nykh s pervym psikhoticheskim epizodom [Prolonged release medications in maintenance therapy of patients with first psychotic episode]. Social'naja i klinicheskaja psihiatrija [Social and Clinical Psychiatry]. 2014; 3: 67-72 (in Russian).

Received May 26.05.2017 Accepted June 26.2017

Ivanov Mikhail V., MD, Prof., Head of the $1^{\text {st }}$ Departnment of Biological Therapy of Mental Patients, St. Petersburg V.M. Bekhterev Psychoneurological Research Institute, St. Petersburg, Russian Federation.

Kosterin Dmitry N., PhD, researcher of the $1^{\text {st }}$ Departnment of Biological Therapy of Mental Patients, St. Petersburg V.M. Bekhterev Psychoneurological Research Institute, St. Petersburg, Russian Federation.

Ivanov Mikhail V., profmikhailivanov@gmail.com 